\title{
Two-site entropy and quantum phase transitions in low-dimensional models
}

\author{
Ö. Legeza and J. Sólyom \\ Research Institute for Solid State Physics and Optics, H-1525 Budapest, P. O. Box 49, Hungary
}

(Dated: August 9, 2021)

\begin{abstract}
We propose a new approach to study quantum phase transitions in low-dimensional lattice models. It is based on studying the von Neumann entropy of two neighboring central sites in a long chain. It is demonstrated that the procedure works equally well for fermionic and spin models, and the two-site entropy is a better indicator of quantum phase transition than calculating gaps, order parameters or the single-site entropy. The method is especially convenient when the density-matrix renormalization-group (DMRG) algorithm is used.

PACS numbers: 71.10.Fd, 71.30.+h, 75.10.Jm
\end{abstract}

The search for ground state and the study of quantum phase transitions (QPTs) is a challenging problem when strongly correlated fermionic or spin systems are considered. Since exactly solvable models are rare, in most cases the relevant part of the excitation spectrum, the order parameters characterizing the various phases, or eventually susceptibilities are determined numerically on finite chains and their thermodynamic limit is determined using the standard finite-size scaling method. Unfortunately in several cases no definite conclusions can be drawn even if the calculations are performed on rather long chains.

In this letter, we propose a new approach to detect QPTs and to locate the quantum critical point in lowdimensional spin or fermionic models. It is based on studying the behavior of the von Neumann entropy of two neighboring sites in the middle of a long chain, which can be defined both for fermionic and spin models, and can be especially easily implemented when the densitymatrix renormalization-group (DMRG) algorithm [1] is used.

The method is closely related to concepts in quantum information theory, which recently have attracted great attention in relation to QPTs. Wu et al. 2] have shown that quite generally QPTs are signalled by a discontinuity in some measure of entanglement in the quantum system. One such measure is the concurrence 3 . which has been used by a number of authors [4, 5, 6, 6, 7, , , 9, 10] in their study of spin models. Since the concurrence is defined for spin- $1 / 2$ systems only, for higher spins or fermionic models another measure of entanglement is needed.

The local measure of entanglement, the one-site entropy, which is obtained from the reduced density matrix $\rho_{i}$ at site $i$, has been proposed by Zanardi [11] and Gu et al. 12 to identify QPTs. Contrary to their expectation, in many cases, this quantity turns out to be insensitive to QPT. As an example let us consider the most general isotropic spin-1 chain model described by the Hamiltonian

$$
\mathcal{H}=\sum_{i}\left[\cos \theta\left(\boldsymbol{S}_{i} \cdot \boldsymbol{S}_{i+1}\right)+\sin \theta\left(\boldsymbol{S}_{i} \cdot \boldsymbol{S}_{i+1}\right)^{2}\right] .
$$

In $1 \mathrm{D}$, the model can be solved exactly at $\theta= \pm \pi / 4$ and $\theta= \pm 3 \pi / 4$, and is known to have at least four different phases [13]. The ground state is ferromagnetic for $\theta<-3 \pi / 4$ and $\theta>\pi / 2$, while in between the integrable points separate the Haldane phase, which exists in the range $-\pi / 4<\theta<\pi / 4$, from the dimerized and the quantum spin nematic phases, respectively. The existence of another phase, the quantum quadrupolar phase [14] near $\theta=-3 \pi / 4$ is not settled yet [15]. These phases and the corresponding QPTs do not show up at all in the local entropy. As can be seen in Fig. 1 the site entropy has the value $s_{i}=\ln 3$ in the whole range $-3 \pi / 4<\theta<\pi / 2$. This is due to the unbroken $\mathrm{SU}(2)$ symmetry in the nonpolarized states. In the totally aligned ferromagnetic state, finite value is obtained for the single-site entropy, because the $S_{\text {tot }}^{z}=0$ component of this state is considered.

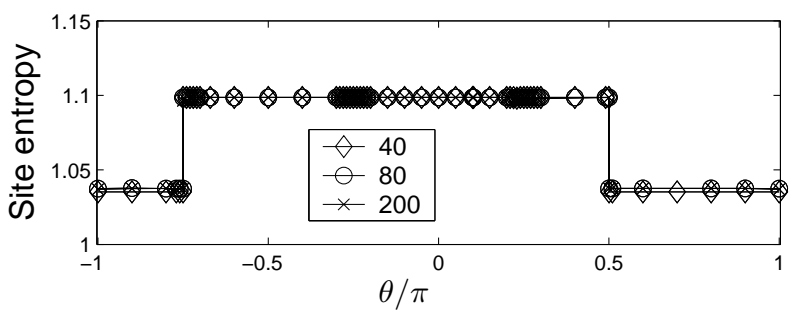

FIG. 1: $\theta$ dependence of the single-site entropy of the central site of the isotropic spin-1 chain, determined for different chain lengths.

The aim of this letter is to point out that a better indicator is obtained if instead of the entropy of a single site, the entropy of two neighboring central sites,

$$
s_{i, i+1}=-\operatorname{Tr} \rho_{i, i+1} \ln \rho_{i, i+1}
$$

is studied, where $\rho_{i, i+1}$ is the reduced density matrix of the two sites. A related quantity has been used in 16. to characterize the interaction between states in quantum chemistry. We will demonstrate that if - in order to avoid boundary effects - sites in the middle of long chains are considered, i.e., for $i=N / 2$ and $i=N / 2+1$, where $N$ is the number of sites in the chain, anomalies are developing in this quantity, and their analysis can be used 
to detect quantum phase transitions in low-dimensional lattice models. This procedure is especially convenient when a dimerization transition is studied. In this case, namely, the difference of two-site entropies on neighboring sites in the center of the chain,

$$
D_{s}(i)=s_{i+1, i+2}-s_{i, i+1}, \quad i=N / 2
$$

is finite, but the method works for other types of transitions, as well.

As usual in the DMRG approach, we considered open chains. The numerical calculations were performed on finite chains up to 400 lattice sites using the dynamic block-state selection (DBSS) approach [17]. The threshold value of the quantum information loss $\chi$ has been set to $10^{-8}$. Similar anomalies appear in the two-site entropy when periodic boundary condition is used, except that $D_{s}$ is meaningless in this case.

As a first example the results obtained for the biliniearbiquadratic model are shown in Fig. 2 The upper panel shows $s_{i, i+1}$ for $i=N / 2$ and $i=N / 2+1$, while $D_{s}(N / 2)$ is displayed in the lower panel.
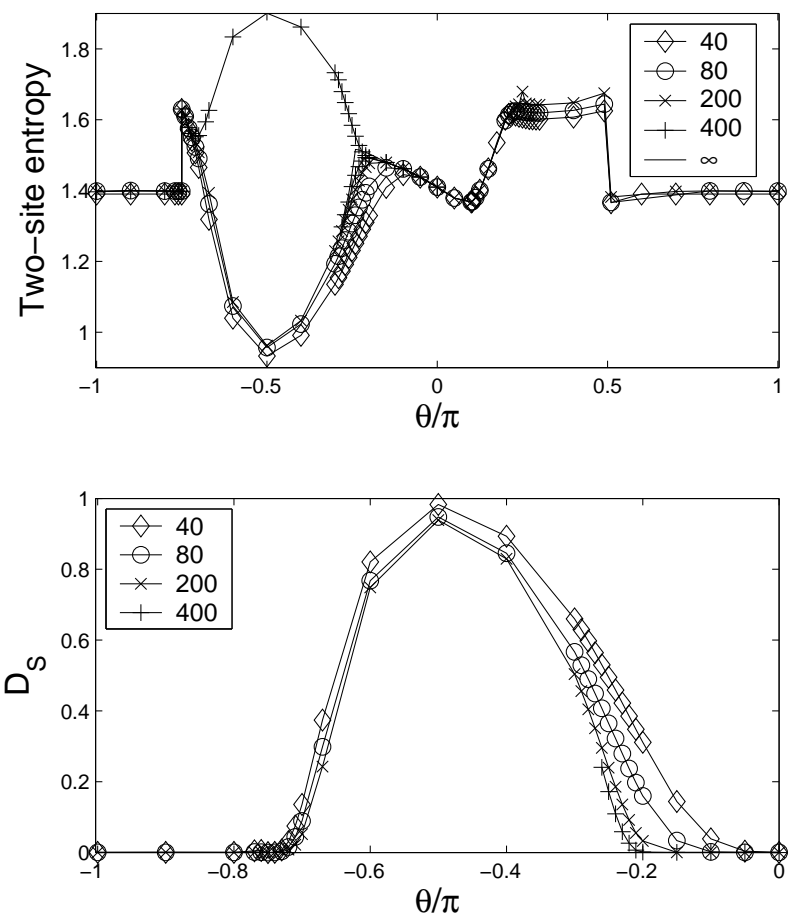

FIG. 2: (a) $\theta$ dependence of the two-site entropy $s_{i, i+1}$ for $i=N / 2$ and $i=N / 2+1$ of the isotropic spin- 1 chain for different chain lengths. The results for $i=N / 2$ are displayed for $N=400$ only. (b) Dimerization of the two-site entropy.

In the range $-3 \pi / 4<\theta<\pi / 2$, one can clearly distinguish three regimes. The most pronounced feature is that the two-site entropy is strongly dimerized for $-3 \pi / 4<\theta<-\pi / 4$, which reflects the dimerization of the valence-bond structure in this regime. The quantity $s_{i, i+1}$ for $i=N / 2$ and $i=N / 2+1$ measures the entropy of a pair of sites coupled by strong or weak bonds, respectively. The lower panel shows that as longer and longer chains are considered, the boundaries of the dimerized region scale to the integrable points, $\theta=-\pi / 4$ and $-3 \pi / 4$.

In the Haldane phase, where all neighbors are coupled predominantly by one valence bond, as in the AKLT state 18], the two-site entropy is not alternating, its minimum is at the AKLT point. Right at $\theta=\pi / 4$, where the model has $\mathrm{SU}(3)$ symmetry, the two-site entropy is discontinuous, moreover in the thermodynamic limit, a kink appears between the left and right sides of this point, which is a clear indication of the transition from the Haldane phase into the quantum spin-nematic phase.

Having demonstrated the usefulness of the study of the two-site entropy on a model where the quantum critical points were known, we now use this procedure to study the phase diagram of two 1D fermionic models proposed to explain the neutral-ionic (N-I) transition first observed in organic mixed-stack charge-transfer salts 19.

The ionic Hubbard model [20] is defined by the Hamiltonian

$$
\begin{aligned}
\mathcal{H}= & t \sum_{i \sigma}\left(c_{i \sigma}^{\dagger} c_{i+1 \sigma}+c_{i+1 \sigma}^{\dagger} c_{i \sigma}\right) \\
& +U \sum_{i} n_{i \uparrow} n_{i \downarrow}+\frac{\Delta}{2} \sum_{i \sigma}(-1)^{i} n_{i \sigma} .
\end{aligned}
$$

When the number of electrons is exactly equal to the number of sites, the competition between the on-site energy difference $\Delta$ and the Coulomb energy $U$ will determine whether the system is a band insulator $(U<\Delta)$ where both the charge and spin gaps are finite or a correlated Mott insulator $(U>\Delta)$ where only the charge gap is finite. When hopping is negligibly weak, the transition takes place at $U=\Delta$. As was first pointed out by Fabrizio et al. 21], for finite $t$ values the transition between these two states occurs in two steps. The charge gap closes and reopens at a critical value $U_{\mathrm{c}}$, while the spin gap vanishes at a different value $U_{\mathrm{s}}$. The transition at $U=U_{\mathrm{c}}$ is Ising-like while the one at $U_{\mathrm{s}}$ is a KosterlitzThouless transition. A dimerized bond-order phase exists between the two critical values $U_{\mathrm{c}}<U<U_{\mathrm{s}}$.

Since then the model has been studied in detail by several groups 22, 23, 24, 25, 26, 27] using both analytic and numerical methods. Despite the many efforts the controversy concerning the number of transitions was not resolved until recently [23, 26, 27]. The difficulty can be seen from Fig. 31 which shows the dimer order parameter (the energy difference of neighboring bonds) for finite chains with open boundary condition. Using the standard finite-size scaling procedure the vanishing of the dimer order cannot be established with certainty from these data.

Moreover, in contrast to what has been found in the extended Hubbard model [7], the single-site entropy is a rather smooth curve without sharp extremum or discontinuity in the derivative. If, however, the two-site entropy 

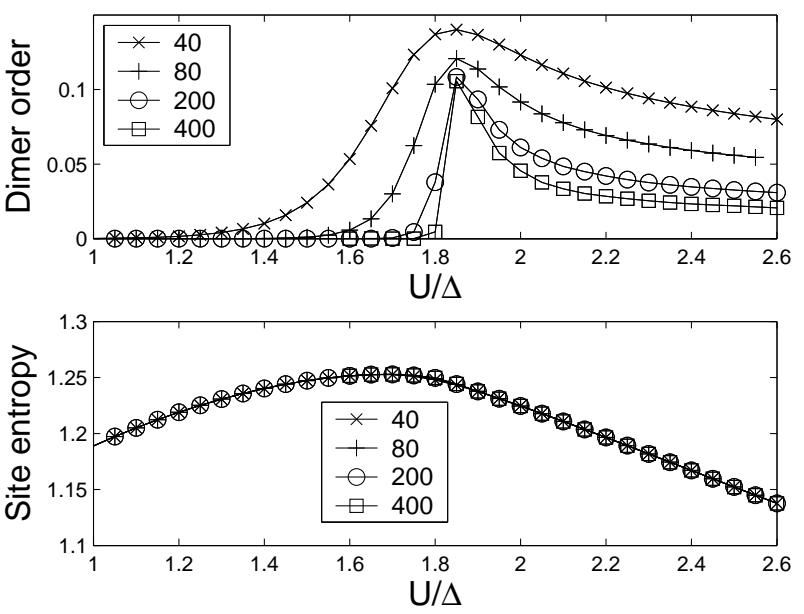

FIG. 3: (a) Dimer order parameter of the ionic Hubbard model for $t / \Delta=1$ for different chain lengths. (b) Entropy of the central site.

is analysed, two transitions can be identified. The results for $t / \Delta=1$ are shown in Fig. [4 The two curves for fixed $N$ correspond to $s_{i, i+1}$ with $i=N / 2$ and $i=N / 2+1$. Similar behavior has been found for other values of $t / \Delta$, too.

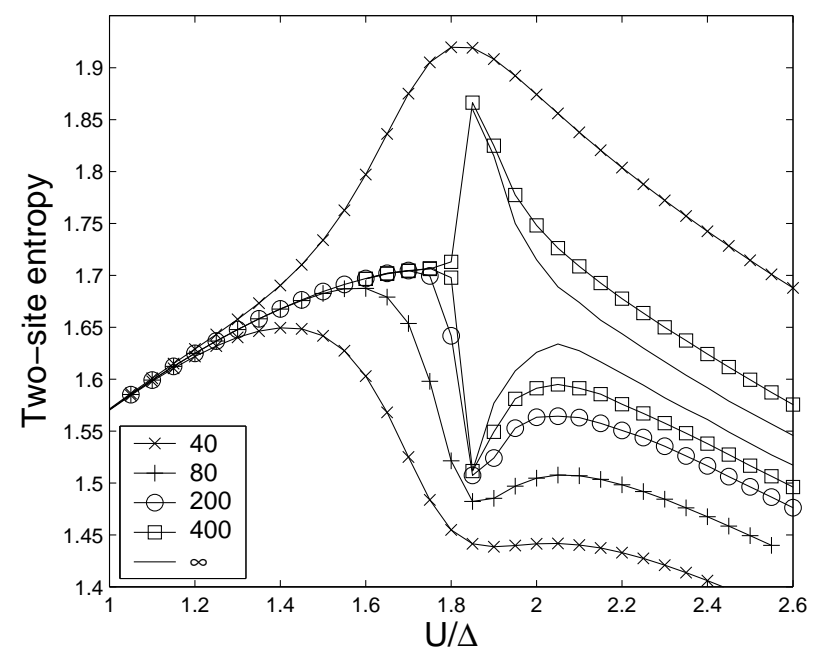

FIG. 4: Two-site entropy $s_{i, i+1}$ for $i=N / 2$ and $i=N / 2+1$ of the ionic Hubbard model for $t / \Delta=1$ for different chain lengths.

The Ising-like transition, the point where the two-site entropy becomes dimerized, can be rather well located. On the other hand the Kosterlitz-Thouless transition at larger values of the Coulomb coupling, where the dimerization disappears again, cannot be established with certainty by looking at $D_{s}$ alone. Standard finite-size scaling does not give a vanishing $D_{s}$. However, the maxima in $s_{i, i+1}$ for $i=N / 2+1$ develop into a cusp in the thermodynamic limit, and this should be attributed to the second transition.

As a next example we have considered another model proposed to describe the neutral-ionic transition [28]. In this model donor molecules with ionization energy $I$ and acceptor molecules with electron affinity $A$, respectively alternate. If the energies are measured with respect to the neutral state, in which the donors are doubly occupied and the acceptors are empty, the molecular levels are described by the Hamiltonian

$$
\begin{aligned}
\mathcal{H}_{0}= & I \sum_{i}\left(2-n_{2 i}\right)+U_{\mathrm{D}} \sum_{i}\left(1-n_{2 i, \uparrow}\right)\left(1-n_{2 i, \downarrow}\right) \\
& -A \sum_{i} n_{2 i-1}+U_{A} \sum_{i} n_{2 i-1, \uparrow} n_{2 i-1, \downarrow}
\end{aligned}
$$

and the hopping, the charge transfer between neighboring donors and acceptors is given by

$$
\begin{aligned}
\mathcal{H}_{\mathrm{CT}}= & t \sum_{i \sigma}\left[c_{2 i \sigma}^{\dagger}\left(c_{2 i-1, \sigma}+c_{2 i+1, \sigma}\right)\right. \\
& \left.+\left(c_{2 i-1, \sigma}^{\dagger}+c_{2 i+1, \sigma}^{\dagger}\right) c_{2 i, \sigma}\right] .
\end{aligned}
$$

In realistic charge-transfer salts the on-site Coulomb energy is presumably the largest energy, so it is reasonable to assume that its unique role is to forbid doubly ionized (empty) donors and doubly ionized (doubly occupied) acceptors. The transition between the neutral and ionic phases is driven by the intersite Coulomb coupling,

$$
\mathcal{H}_{\mathrm{C}}=-V \sum_{i}\left(2-n_{2 i}\right)\left(n_{2 i-1}+n_{2 i+1}\right) .
$$

The relevant parameter is $V / \Delta$, where $\Delta=\frac{1}{2}(I-A)$.

This problem has been studied by exact diagonalization on relatively short chains 28] as well as by valencebond techniques [29]. For small transfer integral the model was found to exhibit a first-order phase transition form a neutral to an ionic phase as a function of $V / \Delta$. For $t / \Delta$ larger than 0.3 the transition changes character and a second-order phase transition has been reported. Our numerical result for the site entropy for $t / \Delta=1$ is shown in Fig. [5 A cusp is clearly seen in the one-site entropy at $V / \Delta \simeq 1.42$, indicating a single second-order transition, in agreement with earlier expectations.

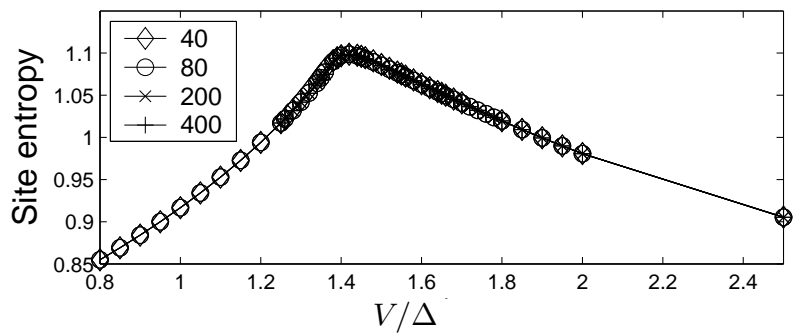

FIG. 5: Single-site entropy of the donor-acceptor model for $t / \Delta=1$ for different chain lengths.

Contrary to this the two-site entropy shown in Fig. [6 exhibits the same behavior as the ionic Hubbard model. 
In fact, as will be shown in a separate paper, the two models are limiting cases of a unified model of neutralionic transition. Although, as for the ionic Hubbard model, it is not easy to locate the vanishing of dimerization when $D_{s}$ alone is considered, the peak of $s_{i, i+1}$ for $i=N / 2+1$ produces a cusp in the thermodynamic limit, indicating a second transition. For small transfer integral, $t / \Delta<0.3$, the two transition points coalesce, both the one and two-site entropies exhibit a sharp jump, the transition is of first order.

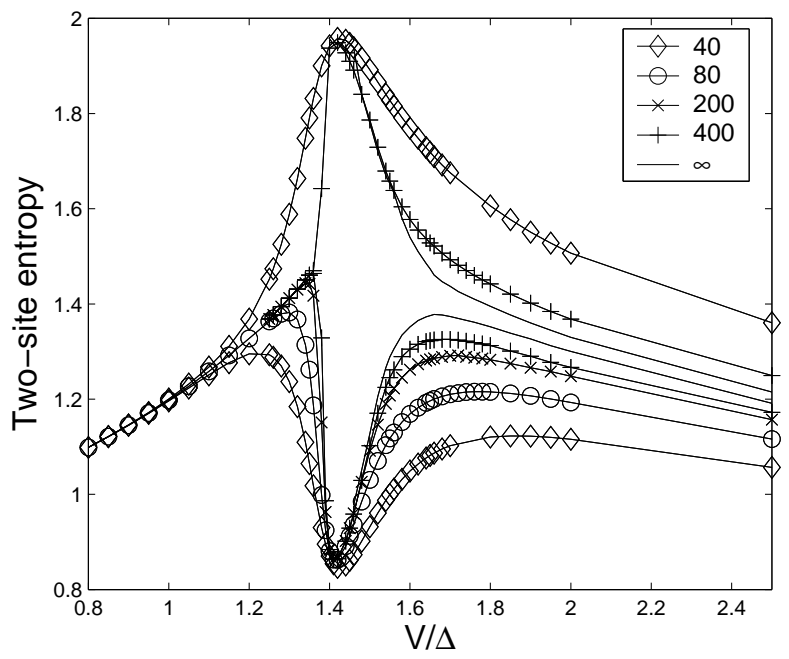

FIG. 6: Two-site entropy of the donor-acceptor model for $t / \Delta=1$ for different chain lengths.

In conclusion, we have shown that the entropy of two central sites in a long open spin or fermionic chain, which can be easily calculated in the density-matrix renormalization-group procedure provides us with extra information not contained in the single-site entropy. The two-site entropy displays maxima or break points at QPT even in such cases when it is difficult to establish the opening or closing of a gap, or the vanishing of an order parameter, or when the single-site entropy is featureless since it is insensitive to the breaking of symmetry that distinguishes the two phases. Using this procedure we have demonstrated that the ionic Hubbard model and the donor-acceptor model do in fact have two phase transitions.

The procedure can be easily extended to considering the entropy of not just two-sites, but that of a longer segment, whose length dependence is different whether the model is critical or not [30]. Fig. 7] shows the entropy of the block of $N / 2$ and $N / 2+1$ sites of an $N=200$ chain for the bilinear-biquadratic $S=1$ model. The dimerization appears in the block entropy, as well, and the anomalies whose location scales to the transition points $\theta= \pm \pi / 4$ are more pronounced than in the two-site entropy. We have learned, after the submission of our paper, that the study of the block-block entanglement in connection with quantum phase transition has been proposed by Deng et al. 31]. Our experience shows, however, that calculating the block entropy with the same accuracy as that of two sites is a numerically much more demanding task.

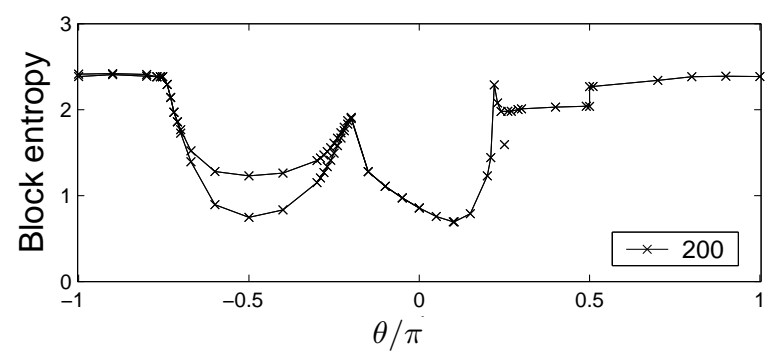

FIG. 7: Entropy of blocks of $N / 2$ and $N / 2+1$ sites of the bilinear-biquadratic $S=1$ model for a chain with $N=200$ sites.

This research was supported in part by the Hungarian Research Fund (OTKA) Grants No. T043330 and F046356. The authors acknowledge computational support from Dynaflex Ltd under Grant No. IgB-32. Ö. L. was also supported by the János Bolyai scholarship.

[1] S.R. White, Phys. Rev. Lett. 69, 2863 (1992); Phys. Rev. B 48, 10345 (1993).

[2] L.-A. Wu, M.S. Sarandy, and D.A. Lidar, Phys. Rev. Lett. 93, 250404 (2004).

[3] W.K. Wootters, Phys. Rev. Lett. 80, 2245 (1998).

[4] T.J. Osborne and M.A. Nielsen, Phys. Rev. A 66, 32110 (2002).

[5] A. Osterloh, L. Amico, G. Falci, and R. Fazio, Nature 416, 608 (2002).

[6] O.F. Syljuåsen, Phys. Rev. A 68, 60301(R) (2003).

[7] S.-J. Gu, H.-Q. Lin, and Y.-Q. Li, Phys. Rev. A 68, 42330 (2003).

[8] J. Vidal, G. Palacios, and R. Mosseri, Phys. Rev. A 69, 022107 (2004); J. Vidal, R. Mosseri, and J. Dukelsky, ibid. 69, 054101 (2004).

[9] T. Roscilde, P. Verrucchi, A. Fubini, S. Haas, and V. Tognetti, Phys. Rev. Lett. 93, 167203 (2004); 94, 147208 (2005).

[10] M.-F. Yang, Phys. Rev. A. 71, 30302(R) (2005).

[11] P. Zanardi, Phys. Rev. A 65, 42101 (2002).

[12] S.-J. Gu, S.-S. Deng, Y.-Q. Li, and H.-Q. Lin, Phys. Rev. Lett. 93, 86402 (2004).

[13] For its phase diagram see G. Fáth and J. Sólyom, Phys. Rev. B 44, 11836 (1991); 47, 872 (1993); 51, 3620 (1995).

[14] A.V. Chubukov, J. Phys. Condens. Matter 2, 1593 (1990); Phys. Rev. B 43, 3337 (1991); A. Läuchli, G. Schmid, and T. Trebst, cond-mat/0311082 (2003).

[15] K. Buchta, G. Fáth, Ö. Legeza and J. Sólyom, Phys. Rev. B 72, 054433 (2005).

[16] J. Rissler, R.M. Noack, and S.R. White, arXiv:cond-mat/0508524 (2005).

[17] O. Legeza, J. Röder, and B.A. Hess, Phys. Rev. B 67, 125114 (2003); Ö. Legeza and J. Sólyom, Phys. Rev. B 70, 205118 (2004). 
[18] I. Affleck, T. Kennedy, E. Lieb, and H. Tasaki, Phys. Rev. Lett. 59, 799 (1987).

[19] J.B. Torrance, J.E. Vazquez, J.J. Mayerle, and V.Y. Lee, Phys. Rev. Lett. 46, 253 (1981).

[20] J. Hubbard and J.B. Torrance, Phys. Rev. Lett 47, 1750 (1981).

[21] M. Fabrizio, A.O. Gogolin, and A.A. Nersesyan, Phys. Rev. Lett. 83, 2014 (1999); Nucl. Phys. B 580, 647 (2000).

[22] A.P. Kampf, M. Sekania, G.I. Japaridze, and P. Brune, J. Phys.: Condens. Matter 15, 5895 (2003).

[23] S.R. Manmana, V. Meden, R.M. Noack, and K. Schönhammer, Phys. Rev. B 70, 155115 (2004).

[24] Z.G. Soos, S.A. Bewick, A. Peri, and A. Painelli, J.
Chem. Phys. 120, 6712 (2004).

[25] A.A. Aligia and C.D. Batista, cond-mat/0412026 (2004).

[26] L. Tincani, R. Noack, and D. Baeriswyl (unpublished).

[27] H. Otsuka and M. Nakamura, Phys. Rev. B 71, 155105 (2005).

[28] B. Horovitz and J. Sólyom, Phys. Rev. B 35, 7081 (1987).

[29] A. Painelli and A. Girlando, Phys. Rev. B 37, 5748 (1988).

[30] G. Vidal, J.I. Latorre, E. Rico, and A. Kitaev, Phys. Rev. Lett. 90, 227902 (2003).

[31] S.-S. Deng, S.-J. Gu, and H.-Q. Lin, arXiv:quant-ph/0511103 (2005). 